\title{
Competencias psicológicas en el personal de enfermería para el tratamiento de pacientes con COVID-19
}

\section{Psychological competencies in nursing staff for the treatment of patients with COVID-19}

Noralma Katherine Jaime-Hernández norahernandez10@hotmail.es

Universidad Estatal del Sur de Manabí, Jipijapa

Ecuador

https://orcid.org/0000-0001-9292-7337

Silvia Cristina Peña-Parrales silviapenaparrales@gmail.com

Universidad Estatal del Sur de Manabí, Jipijapa

Ecuador

https://orcid.org/0000-0002-7157-4648

Johana Alejandra García-Luna

alejandragarcialu@gmail.com

Universidad Estatal del Sur de Manabí, Jipijapa

Ecuador

https://orcid.org/0000-0002-6052-3618

Recibido: 30 de julio de 2021

Aprobado: 15 de octubre de 2021 


\title{
RESUMEN
}

El mundo entero sufre una gran enfermedad producto de un virus mundial. La siguiente investigación presentó como objetivo general analizar las competencias psicológicas en el personal de enfermería para el tratamiento de pacientes con COVID-19. Está enmarcada en un diseño documental-bibliográfico, desde el paradigma cuantitativo, ya que se analiza y estudia una serie de datos obtenidos relacionados a las competencias psicológicas en el personal de enfermería para el tratamiento de pacientes con COVID-19, la población se constituyó por revistas, trabajos arbitrados lo que fundamentó el contenido teórico. Fue empleada la técnica de análisis de contenido, la cual consintió en estudiar y considerar las, características y dimensiones, organizando la relación de las mismas. Se concluye que el talento humano de salud, y en este caso en de enfermería acaece doble vulnerabilidad, no obstante, su seguridad psicológica, no han realizado el apoyo suficiente con gestiones determinadas que resguarden su misión y vocación.

Descriptores: Personal paramédico; tratamiento médico; salud pública. (Tesauro UNESCO)

\begin{abstract}
The whole world suffers from a great disease caused by a global virus. The following research presented as a general objective to analyze the psychological competencies in nursing personnel for the treatment of patients with COVID-19. It is framed in a documentary-bibliographic design, from the quantitative paradigm, since a series of data obtained related to the psychological competencies in nursing personnel is analyzed and studied for the treatment of patients with COVID-19, the population was constituted by magazines, refereed works which supported the theoretical content. The content analysis technique was used, which allowed to study and consider the characteristics and dimensions, organizing their relationship. It is concluded that human talent in health, and in this case in nursing, there is a double vulnerability, however, their psychological security, they have not provided sufficient support with certain steps that protect their mission and vocation.
\end{abstract}

Descriptors: Paramedic personnel; medical treatment; public health. (UNESCO Thesaurus) 


\section{INTRODUCCIÓN}

El sistema de salud cuenta con un talento humano de mucha importancia como lo es el personal de enfermería, quienes brindan un gran apoyo al cuerpo de médicos y especialistas que forman parte de los diferentes centros propiciadores de salud. La profesionalización y actualización del grupo de enfermería es de gran relevancia, para lograr los objetivos y cumplir con eficiencia y eficacia a la atención con mística, dedicación y compromiso, de los diferentes pacientes que asisten a las sedes asistenciales en busca de mejorar su salud. La enfermería, al igual que otras profesiones de la salud, debe asegurarse de que se consideran justamente los valores de cada persona y que no se abuse de sus derechos y privilegios. (Zabalegui Yárnoz, 2003, p.18)

En tal sentido, muchos de los pacientes llegan con muchas inquietudes y requerimientos tanto físicos, como emocionales, para lo cual las enfermeras(os) deben están preparados mediante técnicas y estrategias para la atención efectiva de las personas. Sin embargo, muchas veces el quehacer diario, tanto en el lugar de trabajo, como en su hogar provocan algunas alteraciones en su estado anímico y psicológico. Los cuales deben ser auto controlados, hasta llegar a una crisis emocional que puede desencadenar en hechos de enfrentamientos, mala praxis e inestabilidad psicológica.

En la actualidad, el personal de salud se enfrenta a una crisis producto de una pandemia mundial, en enero de 2020, la Organización Mundial de la Salud (OMS) declaró la pandemia mundial COVID-19, como una emergencia internacional. Al respecto, Lino-Solís, et al. (2021) plantea:

Uno de los mayores retos del personal de enfermería en el periodo de pandemia por COVID-19, ha sido el relacionado a la salud mental, sobre todo en los inicios de la enfermedad, donde no existía información convincente para tratar sanitariamente el virus, existiendo incertidumbre del modo de contagio, siendo así que las enfermeras se encuentran en primera línea de acción clínica, se genera estrés y conflictos emocionales, por temor al contagio, así como transmitirlos a sus familiares con los cuales conviven.(p.35) 
A pesar de los temores, por enfrentarse a una enfermedad desconocida los profesionales de la enfermaría siguen cumpliendo con su labor, en muchos casos sin contar con el apoyo necesario para la protección e integridad de su salud, lo mueve su vocación de servicio, la/el enfermera/o está en constante presión de trabajo, enfrentada al dolor, sufrimiento, desesperanza, muerte, desconsuelo, impotencia y angustia; además, la falta de trabajadores que puedan abarcar a cabalidad los requerimientos de la población y de las instituciones. (Següel Palma, Valenzuela Süazo y Sanhueza Alvarado,2015, p.12).

En este sentido, el talento humano de la salud, se enfrenta a la pandemia con una triple concepción por cuanto debe pensar el resguardo de sus seres queridos, de los pacientes y de sí mismo. Por lo tanto, es recomendable el apoyo institucional para garantizar los servicios y una constante preparación ante una enfermedad relativamente nueva, en concordancia con los autores Amaya Córdova, et al, (2021):

Los principales factores a tener en cuenta para gestionar favorablemente el servicio de enfermería en período COVID-19, se relacionan con el aprendizaje permanente y sobre la marcha de la pandemia, trabajo en equipo, equipamiento y protección personal, cuidado de la salud mental. (p.29)

Sin duda alguna, el apoyo del personal de salud por la lucha contra la pandemia es relevante ya que se trata de salvar vidas aun a costa de la pérdida de la propia. Pero es alarmante el número de personal de la salud que han fallecido a causa de contagio por el COVID-19, al respeto Lahite Savón, Céspedes Pereña y Maslen Bonnane (2020), indican las siguientes estadísticas:

Durante la COVID-19 se han infectado cerca de 90 mil enfermeras y han fallecido más de 200 por esta causa, y el riesgo persiste, en tanto las enfermeras y los enfermeros están en todos los escenarios donde se afronta la COVID-19, por lo que las organizaciones internacionales de Enfermería demandan de la realización de acciones informativas para estas profesionales con énfasis en cómo actuar en los diversos escenarios. (p.496)

De acuerdo a lo delineado se presenta como objetivo general de la investigación analizar las Competencias psicológicas en el personal de enfermería para el tratamiento de pacientes con COVID-19. 


\section{METODOLOGÍA}

La presente investigación está enmarcada en un diseño documental-bibliográfico, desde el paradigma cuantitativo, ya que se analiza y estudia una serie de datos obtenidos relacionados a las competencias psicológicas en el personal de enfermería para el tratamiento de pacientes con COVID-19. La indagación, recuperación, el examen crítico y la interpretación de las investigaciones registradas por otros investigadores en fuentes impresas y bibliográficas que apoyan la investigación documental, la población está constituida por revistas, trabajos arbitrados lo que fundamentó el contenido teórico. Por otro lado, la técnica de análisis de contenido, consintió en estudiar y considerar las, características y dimensiones, organizando la relación de las mismas, lo que permitió comparar y contrastar con la realidad y cumplir con el objetivo de la investigación.

\section{RESULTADOS}

Luego de la revisión bibliográfica mediante el análisis documental se muestran a continuación los resultados obtenidos:

En el siguiente cuadro 1, se describen algunas de las situaciones o factores por las cuales el personal de la salud experimenta en su jornada diaria y puede ocasionar algunos riesgos profesionales de no ser atendidos a tiempo.

\section{Cuadro1.}

Factores profesional.

\begin{tabular}{|l|l|}
\hline \multicolumn{1}{|c|}{ Autor(es) } & \multicolumn{1}{|c|}{ Causas/tipo } \\
\hline $\begin{array}{l}\text { Loyola da Silva, T., de Medeiros Pinheiro } \\
\text { Fernandes, A., Brito do O'Silva, C., de Mesquita X., } \\
\text { Suênia S., y Bezerra de Macedo, E. (2021). }\end{array}$ & $\begin{array}{l}\text { La presión sobre los profesionales de salud, por lo } \\
\text { tanto, aumenta, además de los riesgos de } \\
\text { contaminación, el estrés físico y emocional. Ante } \\
\text { esto, por considerar la enfermería como parte } \\
\text { integrante fundamental del equipo multiprofesional } \\
\text { de salud. (p.504) }\end{array}$ \\
\hline $\begin{array}{l}\text { Pérez Toríz, J., Báez Hernández, F., Flores Merlo, } \\
\text { M., Nava Navarro, V., Morales Nieto, A., y Zenteno } \\
\text { López, M. (2020) }\end{array}$ & $\begin{array}{l}\text { El personal de enfermería como elementos clave } \\
\text { para la contención (prevención, control y } \\
\text { rehabilitación) de esta enfermedad, han sacrificado } \\
\text { su propio bienestar, debido entre otras cosas, a la }\end{array}$ \\
\hline
\end{tabular}


Anzules Guerra, J., Véliz Zevallos, I., Vinces Centeno, M., \& Menéndez Pin, T. (2019) presión asistencial y al miedo a contagiarse que, sumado al conocimiento de la muerte de sus compañeros, han comenzado a causarles una gran presión psicoemocional. (p.4)

El síndrome de burnout es una entidad psicológica negativa secundaria al estrés interpersonal crónico laboral, que aparece cuando la capacidad de afrontamiento falla, dejando notar la falta de energía de la persona.

\section{Elaboración: Los autores.}

En el cuadro número 2, se muestran algunas competencias apoyadas por el campo de la psicología, que se pueden desarrollar con el objeto de contrarrestar el desequilibrio que se puedan presentar por causa de la pandemia.

\section{Cuadro 2.}

Competencias.

\begin{tabular}{|c|c|c|}
\hline Autore(s) & Investigación & Desarrollo \\
\hline $\begin{array}{l}\text { Gutiérrez Álvarez, A., } \\
\text { Cruz Almaguer, A., y } \\
\text { Zaldívar } \quad \text { Santos, } \\
\text { E.(2020) }\end{array}$ & $\begin{array}{l}\text { Gestión de seguridad } \\
\text { psicológica del personal } \\
\text { sanitario en situaciones } \\
\text { de emergencia por } \\
\text { COVID-19 en el contexto } \\
\text { hospitalario o de } \\
\text { aislamiento. }\end{array}$ & $\begin{array}{l}\text { Las autoras entienden que la seguridad psicológica } \\
\text { es un componente de la bioseguridad que garantiza } \\
\text { la integridad, estabilidad y equilibrio psíquico } \\
\text { (resilencia) del personal encargado del manejo de } \\
\text { pacientes en situaciones de emergencia sanitaria, y } \\
\text { constituye la premisa fundamental para el } \\
\text { cumplimiento con calidad de las estrategias que se } \\
\text { desarrollen en esas circunstancias. Situación de } \\
\text { alarma, que requieren la intervención: } \\
\text { - Comentarios sobre el estado de agotamiento físico. } \\
\text { - Actitud reservada para el cumplimiento de algunas } \\
\text { acciones que requieren mayor nivel de riesgo. } \\
\text {-Necesidad constante de realizar llamadas } \\
\text { telefónicas a su familia o amigos. } \\
\text { - Búsqueda frecuente de información sobre la } \\
\text { enfermedad en diferentes fuentes y cierta credulidad } \\
\text { con noticias poco realistas. } \\
\text { - Referencia a muchos problemas que ha dejado en } \\
\text { su casa o familia. } \\
\text {-Cambio en la calidad del rapport que anteriormente } \\
\text { lograba establecer con el paciente o en la relación } \\
\text { entre los compañeros. }\end{array}$ \\
\hline
\end{tabular}




\begin{tabular}{|l|l|l|}
\hline $\begin{array}{l}\text { Rodríguez Rodríguez, } \\
\text { T., Fonseca Fernández, } \\
\text { M., }\end{array}$ & $\begin{array}{l}\text { Protocolo de actuación } \\
\text { psicológica ante la } \\
\text { COVID-19 en centros }\end{array}$ & $\begin{array}{l}\text { La intervención psicológica en situaciones de crisis } \\
\text { se orienta hacia la expresión de los sentimientos y } \\
\text { experiencias internas de la persona en relación con } \\
\text { el evento o eventos que provocaron el desajuste, } \\
\text { facilita la elaboración del duelo ante la pérdida, en } \\
\text { algunas ocasiones de bienes materiales, y en otras } \\
\text { Angulo, L. (2020) }\end{array}$ \\
$\begin{array}{l}\text { asistenciales. } \\
\text { la de un ser querido. (p.369) }\end{array}$ \\
$\begin{array}{l}\text { Ferreira do Nascimento, Yuri Hattori, T., y } \\
\text { Pereira Terças-Trettel, } \\
\text { A. (2020). }\end{array}$ & $\begin{array}{l}\text { Dificultades y temores } \\
\text { de las enfermeras que } \\
\text { enfrentan la pandemia } \\
\text { de COVID-19 en Brasil }\end{array}$ & $\begin{array}{l}\text { Estos profesionales todavía necesitan apoyo, } \\
\text { protección y condiciones de trabajo adecuados, y } \\
\text { estímulo para lograr mantener la salud mental, en } \\
\text { medio de las sensaciones de la aflicción, la } \\
\text { imprevisibilidad y el agotamiento. }\end{array}$ \\
\hline
\end{tabular}

Elaboración: Los autores.

\section{CONCLUSIÓN}

El talento humano del área de la salud ha demostrado durante la pandemia que tienen vocación de servicio, al brindar la atención primaria de salud y las orientaciones correspondiente a los pacientes que acuden a los centros asistenciales. El personal de enfermería es un pilar de suma importancia para la distribución y apoyo a la gestión de salud. El escenario de la pandemia por COVID-19, deja a su paso una huella inapreciable en el interior de las vidas de las personas y por ende de la sociedad. Las devastaciones a nivel psicológico son visibles desde las acciones iniciales de prevención que orina la imposición del aislamiento y las cuarentenas. El talento humano de salud, y en este caso en de enfermería acaece doble vulnerabilidad, no obstante, su seguridad psicológica, no han realizado el apoyo suficiente con gestiones determinadas que resguarden su misión.

\section{REFERENCIAS CONSULTADAS}

Amaya Córdova, R., López Orrala, A., Lara Gorozabel, M., y Toala Pincay, K. (2021). Gestión de los servicios de Enfermería frente al COVID-19. Revista Arbitrada Interdisciplinaria de Ciencias de la Salud. Salud y Vida, 5(10), 24-32. doi:http://dx.doi.org/10.35381/s.v.v5i10.1402 
Anzules Guerra, J., Véliz Zevallos, I., Vinces Centeno, M., y Menéndez Pin, T. (2019). Síndrome de burnout: Un riesgo psicosocial en la atención médica. Revista Arbitrada Interdisciplinaria de Ciencias de la Salud. Salud y Vida, 3(6), 839-859. doi:http://dx.doi.org/10.35381/s.v.v3i6.393

Ferreira do Nascimento, V., Yuri Hattori, T., y Pereira Terças-Trettel, A. (2020). Dificultades $y$ temores de las enfermeras que enfrentan la pandemia de COVID-19 en Brasil. Humanidades Médicas, 20(2), 312-333. Recuperado de: https://n9.cl/0d3nb

Gutiérrez Álvarez, A., Cruz Almaguer, A., y Zaldivar Santos, E. (2020). Gestión de seguridad psicológica del personal sanitario en situaciones de emergencia por COVID-19 en el contexto hospitalario o de aislamiento. Revista Cubana de Enfermería, 36(2),1-20. Recuperado de: https://n9.cl/f0h5x

Lahite Savón, Y., Céspedes Pereña, V., y Maslen Bonnane, M. (2020). El desempeño del personal de Enfermería durante la pandemia de la COVID-19. Revista Información Científica, 99(5), 494-502. Recuperado de: https://n9.cl/0g32s

Lino Solís, T., Pisco Álvarez, S., Delvalle Calderón, G., y Chancay Pionce, A. (2021). Impacto del estrés en la práctica de Enfermería. Revista Arbitrada Interdisciplinaria de Ciencias de la Salud. Salud y Vida, 5(10), 33-41. doi:http://dx.doi.org/10.35381/s.v.v5i10.1403

Loyola da Silva, T., de Medeiros Pinheiro Fernandes, Á., Brito do O'Silva, C., de Mesquita X., Suênia S., y Bezerra de Macedo, E. (2021). El impacto de la pandemia en el rol de la enfermería: una revisión narrativa de la literatura. Enfermería Global, 20(63), 502-543. https://dx.doi.org/10.6018/eglobal.454061

Naciones Unidas (2020) Informes de políticas: La COVID-19 y la necesidad de actuar en relación con la salud mental. Recuperado de: https://n9.cl/h1zam

OMS (2020) Garantizar la seguridad de los trabajadores de la salud para preservar la de los pacientes. Recuperado de: $\underline{\text { https://n9.cl/rvvb }}$

Organización Panamericana de la Salud (2020) Cerca de 570.000 trabajadores de la salud se han infectado y 2.500 han muerto por COVID-19 en las Américas. Recuperado de: https://n9.cl/3mb1f 


\section{CIENCIAMATRIA}

Revista Interdisciplinaria de Humanidades, Educación, Ciencia y Tecnología

Año VII. Vol. VII. N¹. Edición Especial. 2021

Hecho el depósito de ley: pp201602FA4721

ISSN-L: 2542-3029; ISSN: 2610-802X

Universidad Nacional Experimental Francisco de Miranda (UNEFM). Santa Ana de Coro. Venezuela

Noralma Katherine Jaime-Hernández; Silvia Cristina Peña-Parrales; Johana Alejandra García-Luna

Pérez Toríz, J., Báez Hernández, F., Flores Merlo, M., Nava Navarro, V., Morales Nieto, A., y Zenteno López, M. (2020). El significado del cuidado enfermero en el contexto de la pandemia COVID-19. Sanus, 5(16), 1-13.https://doi.org/10.36789/sanus.vi16.236

Rodríguez Rodríguez, T., Fonseca Fernández, M., Valladares González, A., y López Angulo, L. (2020). Protocolo de actuación psicológica ante la COVID-19 en centros asistenciales. Cienfuegos. Cuba. MediSur, 18(3), 368-380. Recuperado de: https://n9.cl/euley

Següel Palma, F.; Valenzuela Süazo, S, y Sanhueza Alvarado, O. (2015). El Trabajo del Profesional de Enfermería: Revisión de la Literatura. Ciencia y enfermería, 21(2), 11 20. https://dx.doi.org/10.4067/S0717-95532015000200002

Zabalegui Yárnoz, A. (2003). El rol del profesional en enfermería. Aquichan, 3(1), 16-20. Recuperado de: https://n9.cl/cbw7h 